\title{
Orientación de la formación del modo de actuación atención médica integral: una estrategia pedagógica desde la teoría de la formación por etapas de las acciones mentales
}

\author{
Orientação da formação do modo de atuação atenção médica integral: \\ uma estratégia pedagógica desde a teoria da formação por etapa das \\ ações mentais
}

Miguel Angel Toledo Méndez ${ }^{1}$

Isaac Iran Cabrera Ruiz ${ }^{2}$

\begin{abstract}
RESUMEM
El artículo propone una estrategia pedagógica para la formación del modo de actuación atención médica integral desde la teoría de la formación por etapas de las acciones mentales. La principal contribución y novedad radica en que posibilita la vinculación de la disciplina Psicología al proceso de formación del modo de actuación atención médica integral, mediante una lógica donde se relacionan invariantes de habilidades y acciones específicas en respuesta a las habilidades profesionales y acciones generalizadas de dispensarización, análisis de la situación de salud, diagnóstico e intervención, que determina los esquemas de bases orientadoras para la creación de las condiciones que intervienen en la formación de las acciones a concretar a través del componente laboral y en interacción con los componentes académico e investigativo. Los resultados forman parte de una investigación realizada entre los años 2016 y 2019 y coordinada entre la Facultad de Medicina de la Universidad de Ciencias Médicas y la Universidad Central "Marta Abreu" de Las Villas, ambas radicadas en Villa Clara, Cuba.
\end{abstract}

Palavras-clave: Orientación. Modo de actuación. Atención médica integral.

\begin{abstract}
RESUMO
$\mathrm{O}$ artigo propõe uma estratégia pedagógica para a formação do modo de atuação da atenção médica integral segundo a teoria da formação por etapas das ações mentais. A principal contribuição e novidade possibilita a vinculação da disciplina Psicologia ao processo da formação e modo de atuação para a atenção médica integral. Adota-se uma lógica onde relacionam-se invariantes de habilidades e ações específicas em resposta às habilidades profissionais e ações gerais da atenção primária da saúde. Essa possibilidade determina os esquemas das bases orientadoras para a criação das condições que promovam a formação das ações a serem concretizadas por meio do componente laboral em interação com os componentes acadêmico e investigativo. Os resultados formam parte de uma investigação realizada entre os anos 2016 e 2019, sob a coordenação da Faculdade de Medicina da Universidade de Ciências Médicas de Villa Clara e da Universidade Central "Marta Abreu" das Villas, Santa Clara, Villa Clara, Cuba.
\end{abstract}

Palavras-chave: Orientação. Modo de Atuação. Atenção médica integral.

\footnotetext{
${ }^{1}$ Licenciado en Psicología, Máster en Ciencias de la Educación y Doctor en Ciencias Pedagógicas. Profesor auxiliar del Departamento de Psicología de la Universidad de Ciencias Médicas de Villa Clara. ORCID: https://orcid.org/0000-0002-9684-4501. E-mail: migueltm@ucm.vcl.sld.cu.

2 Licenciado en Psicología; Máster en Ciencias de la Educación; Doctor en Ciencias Pedagógicas. Profesor Titular del departamento de Psicología de la Facultad de Ciencias Sociales de la Universidad Central "Marta Abreu" de Las Villas. Coordinador de la Maestría en Intervención Psicosocial, y de la Disciplina académica Psicología Social. ORCID: https://orcid.org/0000-00019354-539X. E-mail: isaacicr@uclv.edu.cu.
} 


\section{Introducción}

En el ámbito de la educación superior cubana la vinculación del estudio con el trabajo también se considera una idea rectora fundamental (HORRUITINER SILVA, 2006) y propone la necesidad de vincular lo educativo y lo instructivo con lo desarrollador, para garantizar desde el currículo el dominio de los modos de actuación que aseguran la formación profesional.

Las universidades cubanas se apoyan en una red estable de entidades laborales donde se vinculan los estudiantes para cumplir tareas profesionales; idea generalizada de la experiencia acumulada en el desarrollo de la formación del profesional de la medicina a través del componente laboral que distingue como forma organizativa principal la educación en el trabajo y en interacción con los componentes académico e investigativo. Dicho vínculo se ha ido perfeccionando en su diseño y ejecución, asumiéndose un nuevo y transformador enfoque de lo laboral que deviene modelo de formación universitaria desde el trabajo (HORRUITINER SILVA, 2006).

La formación de médicos en la educación superior cubana, unido a la formación tradicional en los hospitales, se decidió enfatizar en la vinculación del año terminal con los consultorios médicos para la solución de problemas profesionales concernientes a las condiciones de vida, médico-legales, de administración de servicios de salud, docentes e investigativos. Los estudiantes cumplimentan tareas relacionadas con las funciones que precisan diferentes modos de actuación profesional de atención médica integral, docente-educativa, administrativa, de investigación y para situaciones excepcionales, en respuesta al objeto de ofrecer atención calificada a la población en el proceso salud-enfermedad y al encargo de garantizar el mantenimiento de una relación médico-paciente-familia-comunidad estable y continua que engendre compromiso y satisfacción mutuos.

Además se comenzó a generalizar una experiencia similar con los estudiantes del primer año de la carrera, vinculándolos a los policlínicos docentes y a la atención ambulatoria; tipo de educación en el trabajo propia de la atención 
primaria de salud o de la asistencia sanitaria esencial, aunque también se desarrolla en el área hospitalaria.

Durante la atención ambulatoria los estudiantes observan y participan en la aplicación del método científico que posibilita el aprendizaje activo de las formas, métodos y normativas de dicho trabajo; de otra parte permite ejercitar de forma tutoral el método clínico durante la planificación y el desarrollo de la consulta asistencial, tomándose en consideración los cambios en las concepciones de la práctica social de la atención en salud. Sin embargo los resultados inmediatos ponen al descubierto que aún no se satisfacen las necesidades de educación en el trabajo por el insuficiente vínculo de los estudiantes con los servicios de la atención primaria de salud.

A partir del curso académico 2013-2014 se efectuaron ajustes curriculares que ampliaron el fondo de tiempo habilitado desde la educación en el trabajo, para consolidar un proceso formativo contextualizado a la atención médica integral en los servicios de la asistencia sanitaria esencial; lo cual contribuye a crear las condiciones necesarias de apertura a la formación profesional interdisciplinar mediante el vínculo entre los componentes académico, laboral e investigativo.

Sin embargo, la ampliación en el fondo de tiempo habilitado desde la educación en el trabajo para consolidar un proceso formativo contextualizado a la atención médica integral en los servicios de la asistencia sanitaria essencial, es visible sólo en las disciplinas académicas integradoras del perfil de estudio al cual se hace alusión; por lo que se identifican limitaciones en las interacciones entre disciplinas académicas integradoras y no integradoras o derivadoras.

La disciplina académica Psicología constituye una de las áreas de estudio del proceso de formación del médico general en la educación superior cubana. En calidad de disciplina derivadora, que profundiza en la esencia de la cultura psicológica implícita en la lógica de atención médica integral que distingue al modo de actuación esencial de la profesión, integra dos asignaturas, Psicología y Psicología Médica, las cuales han permitido superar las perspectivas modulares tradicionales que resaltaban la atención ambulatoria al hombre enfermo. 
Sin embargo se constatan imprecisiones en el logro de la integración, sistematización y aplicación práctica del contenido de la disciplina Psicología que se relaciona directamente con el proceso de formación del modo de actuación atención médica integral, afectándose su continuidad a través de las diferentes disciplinas académicas que completan la malla curricular de la carrera de Medicina en la educación superior cubana. Esta realidad nos condujo a plantearnos como objetivo proponer una estrategia pedagógica para la formación del modo de actuación atención médica integral desde la disciplina Psicología.

\section{La formación del modo de actuación atención médica integral: una mirada desde la disciplina Psicología}

La interacción del profesional de la medicina con las esferas de actuación del objeto de su profesión está mediatizada, al igual que en los disímiles perfiles de estudio de la educación superior cubana, por la naturaleza de los modos de actuación profesional; estos últimos se desarrollan como proceso y resultado de la formación del profesional a lo largo de su carrera, por lo que determinan qué hace el profesional en el contexto laboral y, sobre todo, cómo lo hace.

En esencia comprendemos los modos de actuación profesional del médico general como el sistema y secuencia de acciones generalizadas que de forma flexible actúan sobre el proceso salud-enfermedad y la atención calificada a la población, con el propósito de transformar y autotransformarse mediante la aprehensión de los valores, las estrategias de aprendizaje y los métodos de trabajo de la atención primaria que caracterizan un desempeño ético-humanista, comprometido con las funciones de atención médica integral, docente-educativa, administrativa, de investigación y para situaciones excepcionales, en respuesta al mantenimiento de una relación médico-paciente-familia-comunidad estable y continua que engendre compromiso y satisfacción mutuos.

En términos didácticos las funciones que precisan diferentes modos de actuación profesional de atención médica integral, docente-educativa, 
administrativa, de investigación y para situaciones excepcionales, se estructuran en habilidades a desplegar en la actividad generalizadora de ofrecer atención calificada a la población en el proceso salud-enfermedad, a partir de una secuencia y sistema de acciones a desarrollar con independencia del contexto de actuación profesional; por lo que cabe señalar que los modos de actuación profesional del médico general conservan una lógica al accionar en la actividad pedagógica y por ende dentro de sus componentes estructurales se encuentran las invariantes de habilidades y las estrategias generales de aprendizaje en el cumplimiento de las funciones y tareas profesionales.

La formación del médico general que distingue el actual perfeccionamiento curricular del sexto plan de estudio de la carrera de Medicina en la educación superior cubana (MINSAP, 2014), identifica la atención médica integral como función rectora y modo de actuación esencial del profesional; desde ambas perspectivas la atención médica integral posibilita la ejecución de tareas de prevención y promoción en salud, caracterización de la salud individual y colectiva de la población, detección de factores de riesgos ambientales, trabajo en equipo con los especialistas y atención médica de urgencia, en respuesta al encargo social de la profesión.

El modo de actuación atención médica integral estructura desde las diferentes disciplinas académicas cuatro actividades procesos que siguen una secuencia y sistema de acciones generalizadas a través del componente laboral y en interacción con los componentes académico e investigativo: dispensarización, análisis de la situación de salud, diagnóstico e intervención (TOLEDO; CABRERA, 2016).

Estas cuatro actividades siguen una secuencia y sistema de acciones generalizadas a concretar desde el modo de actuación atención médica integral; por lo que se estructuran en habilidades a desplegar en la actividad generalizadora de ofrecer atención calificada a la población en el proceso salud-enfermedad (TOLEDO; CABRERA, 2016):

$\checkmark$ Controlar a las personas y familias de la comunidad asignada teniendo en cuenta el registro de riesgos y daños asociados a la determinación de la 
salud en los correspondientes niveles de interacción macrosocial, microsocial e individual.

$\checkmark$ Identificar los problemas de salud que afectan a la población de acuerdo con los perfiles epidemiológicos comunitarios.

$\checkmark$ Percibir las enfermedades, alteraciones y trastornos que desequilibran la salud individual y colectiva en la comunidad asignada a partir de sus signos y síntomas.

$\checkmark$ Solucionar los problemas de salud individual y colectiva en la comunidad asignada tomando en consideración los niveles determinantes de satisfacción de la población ante la calidad de los servicios médicos ofrecidos.

\section{La orientación de la formación del modo de actuación: fundamentos teórico metodológico}

La teoría de la formación por etapas de las acciones mentales enfatiza en cómo la acción se transforma de un plano material a otro ideal manteniendo el mismo contenido objetal. Toda acción humana según Talízina (1985, 1988) constituye un microsistema con funciones que son dividas en las partes orientadora, ejecutiva y de control. La parte orientadora muestra el método, el objetivo y las peculiaridades del objeto hacia el cual se dirigen las acciones. La parte ejecutiva propicia la realización de las acciones en el cumplimiento del objetivo dirigido a la transformación del objeto a través de la actividad y del individuo en sí mismo, sobre la base del método antes orientado. La parte de control hace posible tener informaciones acerca de cómo marcha el cumplimiento de las acciones e introducir las correcciones para reordenar la actividad.

Galperin (1992a) y Talízina $(1985,1988)$ coinciden en que el proceso de formación de las acciones mentales está determinado por el carácter de la parte orientadora de la acción. De esta manera la orientación deviene anticipación de la ejecución y su dirección, base de los procesos psíquicos y cualidades de la acción mental; por lo tanto, orientación del aprendizaje significa representación 
del contenido de la acción y de las condiciones de su cumplimiento en el proceso de formación (GALPERIN, 1992a).

La interacción entre componentes esenciales y niveles de funcionamiento, determina la invariante de estructura de la acción que deriva criterio básico para el diseño de la concepción del modelo de asimilación y de enseñanza propuesto por Talízina (1985, 1988); en el cual los objetivos a declarar en términos de tareas-tipo de la profesión, los contenidos a estructurar sistémicamente como un conjunto de invariantes que revelan la esencia de los conocimientos que se aprenden, así como los métodos y las formas para el aseguramiento de los estados de transición del proceso de asimilación, integran los tres eslabones que permiten organizar por etapas el proceso de formación de las acciones que conforman la actividad del profesional:

1. Etapa de la motivación: interesar a los estudiantes en las tareas concretas en las que se traducen los objetivos ante la solución de los problemas de la profesión y cumplan así con la actividad.

2. Etapa de formación de la base orientadora: descubre ante los estudiantes la representación gráfica donde se esquematiza el contenido de la acción.

3. Etapa de las acciones externas materializadas: comprende la solución de problemas a partir de la representación externa de los conocimientos recién explicados; por lo que pueden emplearse tarjetas de estudio.

4. Etapa que se dedica al trabajo independiente que deben realizar los estudiantes: en ella el profesor garantiza la posibilidad de controlar los pasos que dan los estudiantes para estar seguro que han atravesado correctamente todas las etapas.

5. Etapa de las acciones en forma de lenguaje sin apoyo en acciones externas materializadas: consiste en que los estudiantes expresen todo oralmente.

6. Etapa de la acción mental: se distingue por el carácter automático que adquiere la acción durante su cumplimiento en forma mental. 
Consideramos que la orientación crea las condiciones de formación de las acciones si parte de la determinación de los esquemas de bases orientadoras para la vinculación de la disciplina Psicología al proceso de formación del modo de actuación atención médica integral; de igual modo los esquemas de bases orientadoras establecen relaciones causales de determinación ante las condiciones que se crean para la formación de las acciones. En su elaboración los esquemas de bases orientadoras de la acción integraron conocimientos declarativos y procedimentales; o sea conocimientos donde se transmitan las imágenes de las acciones a ejecutar y de las condiciones de su realización.

La generalización del contenido orientador para la vinculación de la disciplina Psicología al proceso de formación del modo de actuación atención médica integral, está determinada por las invariantes de habilidades en las que se integran núcleos esenciales del conocimiento que exigen la realización de acciones específicas en la práctica profesional.

Lo importante en la asimilación de los puntos de orientación con un carácter generalizado para la vinculación de la disciplina Psicología al proceso de formación del modo de actuación atención médica integral, es que los estudiantes no aprendan de memoria los conocimientos que contienen; sino que logren utilizarlos para la realización de acciones específicas.

Otro aspecto medular es el grado de despliegue de los esquemas de bases orientadoras de las acciones. Se siguió el planteamiento de Talízina (1985, 1988) que toda nueva acción primero tiene que realizarse en una forma desplegada y posteriormente abreviada, hasta que se hagan conscientes todos sus eslabones. Del mismo modo en la asimilación de la base orientadora el grado de independencia que se alcance estará determinado por el tránsito de la acción compartida hacia la acción independiente.

La orientación de las acciones para la vinculación de la disciplina Psicología al proceso de formación del modo de actuación atención médica integral garantiza la cumplimentación de tareas-tipo de la profesión y su adecuación al contenido que se asimila, a los objetivos planteados a la enseñanza y a la esfera que permite incrementar la generalización de las acciones (TALÍZINA, 1985). 
En conclusión la comprensión de las acciones que vinculan a la disciplina Psicología al proceso de formación del modo de actuación atención médica integral, implica una lógica donde se determinan los esquemas de bases orientadoras para la creación de las condiciones que intervienen en la formación de las acciones a concretar a través del componente laboral y en interacción con los componentes académico e investigativo; las condiciones creadas garantizan la cumplimentación de un sistema de tareas dirigido a desarrollar acciones con apoyo en las tarjetas de estudio para la formación del pensamiento teórico y de los métodos de la actividad cognoscitiva, a partir de la aplicación de los procedimientos dados a la solución de tareas en la clase correspondiente.

\section{Estrategia pedagógica}

La estrategia (figura 1) integra fases, direcciones e indicaciones que proyectan la dirección pedagógica que permite la transformación del proceso de formación en cuestión y condiciona el establecimiento de acciones de atención médica integral que contribuye a formar la disciplina Psicología.

Figura 1 - Representación gráfica de la estrategia pedagógica para la formación del modo de actuación atención medica integral.

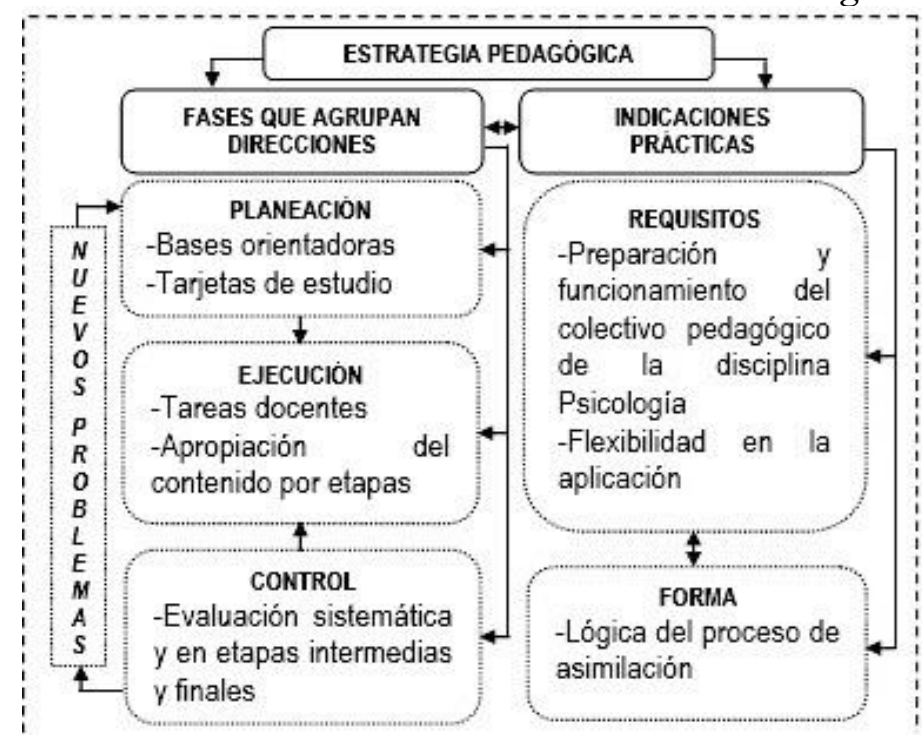

Fonte: Elaboración de los autores (2019) 


\section{Fase de planeación}

Determinación de los esquemas de bases orientadoras de las acciones

1) Esquemas de bases orientadoras de las acciones para el despliegue del proceso de los procesos de dispensarización y análisis de la situación de salud.

$\underline{\text { Conocimiento declarativo }}$

La dispensarización y el análisis de la situación de salud constituyen procesos a desplegar en la atención médica integral desde el contexto de la asistencia sanitaria esencial. La dispensarización hace alusión al registro, agrupamiento y control de las personas y familias asignadas al médico general, al facilitar la observación permanente de la dinámica de las relaciones que se establecen entre individuos y grupos sociales con el objetivo de controlar riesgos y daños a la salud.

El análisis de la situación de salud representa un enfoque multisectorial de la situación de salud poblacional a partir de los perfiles de morbilidad y mortalidad, resultado de la acción de los diferentes actores sociales en la identificación y solución de los problemas existentes.

Conocimiento procedimental

Proceso: Dispensarización

Criterio: La confección de historias clínicas individuales para el establecimiento de las características de los estilos de vida que hacen vulnerables a las personas ante determinados procesos negativos para la salud.

Acciones específicas de la asignatura Psicología: caracterizar las etapas del ciclo vital que se producen en el proceso de desarrollo psicológico del ser humano, sintetizar sus regularidades e interpretar la necesidad de una atención diferenciada respecto a las mismas.

Acciones específicas de la asignatura Psicología Médica: identificar los factores psicosociales que favorecen y/o entorpecen la relación médico-paciente-familiacomunidad, sintetizar sus particularidades ante diversas circunstancias asistenciales y proponer acciones de intervención para el afrontamiento de los factores psicosociales que la entorpecen. 
Criterio: La confección de historias clínicas familiares para el establecimiento de las características de los modos de vida que hacen vulnerables a las familias ante determinados procesos negativos para la salud.

Acciones específicas de la asignatura Psicología: analizar las demandas y exigencias del programa de salud para la atención a la familia, identificar las particularidades de la violencia intrafamiliar y proponer acciones de intervención en su dinámica interna.

Acciones específicas de la asignatura Psicología Médica: identificar los factores que influyen en la afectación de la salud familiar ante el estrés por la amenaza de pérdida de un integrante de la familia, evaluar los factores protectores y proponer acciones de intervención.

Proceso: Análisis de la situación de salud.

Criterios: La coordinación de procesos de registros de observación e interconsultas con otros especialistas, para la detección de los problemas que afectan la salud de la población desde una perspectiva macrosocial; y la confección de fichas epidemiológicas, particularizando en las circunstancias que sitúan a la comunidad en riesgo de enfermar.

Acciones específicas de la asignatura Psicología: analizar los rasgos de los niveles de interacción social que intervienen en la determinación de la salud comunitaria, explorar mediante registros de observación los criterios para el diagnóstico psicosocial del modo de vida en la comunidad y establecer relación causal entre modo de vida y problemas de salud comunitaria.

Acciones específicas de la asignatura Psicología Médica: analizar factores que integran el cuadro interno de enfermedades que afectan la salud comunitaria, observar manifestaciones psicopatológicas y establecer relaciones causales.

2) Esquemas de bases orientadoras de las acciones para el despliegue del proceso de diagnóstico

\section{$\underline{\text { Conocimiento declarativo }}$}

El diagnóstico constituye un proceso a desplegar en la atención médica integral desde el contexto de la asistencia sanitaria esencial, discerniendo sobre la prioridad de los síntomas en función de un diagnóstico certero. 
Proceso: Diagnóstico.

Criterios: La evaluación de individuos, familias, grupos y comunidades, en el proceso salud-enfermedad, abarcando su estado normal y patológico; y la indagación en las explicaciones de las enfermedades, alteraciones y trastornos que desequilibran la salud, partiendo tanto del estudio de las condiciones normales de expresión del comportamiento humano como de la identificación de lesiones cerebrales focales de base.

Acciones específicas de la asignatura Psicología: explorar mediante preguntas el funcionamiento de los procesos psicológicos, caracterizar los componentes estructurales de la personalidad en su desarrollo psicosocial y establecer relaciones causales.

Acciones específicas de la asignatura Psicología Médica: caracterizar los aspectos psicológicos asociados al dolor, explorar mediante la formulación de preguntas las alteraciones psíquicas vinculadas al estrés y establecer relaciones causales.

3) Esquemas de bases orientadoras de las acciones para el despliegue del proceso de intervención

\section{$\underline{\text { Conocimiento declarativo }}$}

La intervención constituye un proceso a desplegar en la atención médica integral desde el contexto de la asistencia sanitaria esencial. El médico general debe afrontar los problemas de salud en la comunidad con fines promocionales, preventivos y de curación-rehabilitación.

\section{$\underline{\text { Conocimiento procedimental }}$}

Proceso: Intervención.

Criterios: La prestación de los servicios de atención médica integral en forma oportuna y continua a la población, con énfasis en la prevención y modificación de factores y conductas de riesgo; y la coordinación de acciones de educación y promoción de la salud y profilaxis en el hombre sano.

Acciones específicas de la asignatura Psicología: explorar mediante registros de observación y formulación de preguntas la existencia de recursos psicológicos que garanticen el conocimiento y afrontamiento de los problemas de salud 
comunitaria, valorar la introducción de acciones de prevención en salud y aplicar las técnicas de educación para la salud.

Acciones específicas de la asignatura Psicología Médica: explorar mediante registros de observación y formulación de preguntas la inexistencia de recursos psicológicos que garanticen el conocimiento y afrontamiento de los problemas de salud comunitaria, confeccionar historias psicosociales que permitan un diagnóstico psicológico certero y establecer una relación psicoterapéutica efectiva. Elaboración de las tarjetas de estudio

Las tarjetas de estudio derivan instrumentos de orientación de acciones en los servicios de la asistencia sanitaria esencial ante el despliegue de los procesos de dispensarización, análisis de la situación de salud, diagnóstico e intervención. Mediante ellas los estudiantes podrán resolver tareas que reflejen casos típicos de aplicación de las acciones dadas a partir de la representación gráfica donde se esquematiza el contenido del material que tienen que asimilar.

1) Tarjetas de estudio para el despliegue del proceso de dispensarización

Criterio: La confección de historias clínicas individuales para el establecimiento de las características de los estilos de vida que hacen vulnerables a las personas ante determinados procesos negativos para la salud.

Asignatura Psicología.

Objetivo: Identificar la sucesión finita de acciones que se requieren para hacer referencia al proceso de desarrollo psicológico del ser humano y las características de las diferentes etapas del ciclo vital, a partir de la confección de historias clínicas individuales.

Inductores para determinar conocimientos esenciales y acciones preparatorias.

Los estudiantes deben precisar el proceso de desarrollo humano y sus etapas evolutivas.

\section{Asignatura Psicología Médica.}

Objetivo: Identificar la sucesión finita de acciones que se requieren para hacer referencia a la relación médico-paciente-familia-comunidad y los factores 
psicosociales asociados al proceso de comunicación en salud, a partir de la confección de historias clínicas individuales.

Inductores para determinar conocimientos esenciales y acciones preparatorias.

Los estudiantes deben precisar respecto al proceso de comunicación en salud y los factores psicosociales que lo favorecen y entorpecen.

Criterio: La confección de historias clínicas familiares para el establecimiento de las características de los modos de vida que hacen vulnerables a las familias ante determinados procesos negativos para la salud.

Asignatura Psicología.

Objetivo: Identificar la sucesión finita de acciones que se requieren para hacer referencia al programa de atención a la salud familiar y las particularidades de la violencia intrafamiliar con su repercusión en la infancia, a partir de la confección de historias clínicas familiares.

Inductores para determinar conocimientos esenciales y acciones preparatorias.

Los estudiantes deben precisar las categorías familia saludable y violencia intrafamiliar:

\section{Asignatura Psicología Médica.}

Objetivo: Identificar la sucesión finita de acciones que se requieren para hacer referencia a los factores que afectan la salud familiar y los efectos del estrés ante la experiencia de amenaza de pérdida de un integrante de la familia, a partir de la confección de historias clínicas familiares.

Inductores para determinar conocimientos esenciales y acciones preparatorias.

Los estudiantes deben precisar aspectos psicosociales con respecto a la amenaza de pérdida de un integrante de la familia.

2) Tarjetas de estudio para el despliegue del proceso de análisis de la situación de salud

Criterios: La coordinación de procesos de registros de observación e interconsultas con otros especialistas, para la detección de los problemas que afectan la salud de la población desde una perspectiva macrosocial; y la confección de fichas epidemiológicas, particularizando en las circunstancias que sitúan a la comunidad en riesgo de enfermar. 


\section{Asignatura Psicología.}

Objetivo: Identificar la sucesión finita de acciones que se requieren para hacer referencia a los niveles de interacción social determinantes de la salud comunitaria y el diagnóstico psicosocial del modo de vida en la comunidad, a partir de la coordinación de registros de observación e interconsultas con otros especialistas y de la confección de fichas epidemiológicas.

Inductores para determinar conocimientos esenciales y acciones preparatorias.

Los estudiantes deben precisar respecto a las variables determinantes de la salud en la comunidad.

\section{Asignatura Psicología Médica.}

Objetivo: Identificar la sucesión finita de acciones que se requieren para hacer referencia a las alteraciones psíquicas que afectan la salud comunitaria y los factores que integran el cuadro interno de la enfermedad, a partir de la coordinación de registros de observación e interconsultas con otros especialistas y de la confección de fichas epidemiológicas.

Inductores para determinar conocimientos esenciales y acciones preparatorias.

Los estudiantes deben precisar el cuadro interno de la enfermedad

3) Tarjetas de estudio para el despliegue del proceso de diagnóstico

Criterios: La evaluación de individuos, familias, grupos y comunidades, en el proceso salud-enfermedad, abarcando su estado normal y patológico; y la indagación en las explicaciones de las enfermedades, alteraciones y trastornos que desequilibran la salud, partiendo tanto del estudio de las condiciones normales de expresión del comportamiento humano como de la identificación de lesiones cerebrales focales de base.

Asignatura Psicología.

Objetivo: Identificar la sucesión finita de acciones que se requieren para hacer referencia al proceso de desarrollo psicológico del ser humano y al funcionamiento de los procesos psíquicos y las características de los componentes estructurales de la personalidad, a partir de la evaluación e indagación en las explicaciones de las enfermedades y trastornos que desequilibran la salud.

Inductores para determinar conocimientos esenciales y acciones preparatorias. 
Los estudiantes deben precisar los procesos psicológicos y los componentes estructurales de la personalidad

\section{Asignatura Psicología Médica.}

Objetivo: Identificar la sucesión finita de acciones que se requieren para hacer referencia a las alteraciones psíquicas vinculadas al estrés y las características de los aspectos psicológicos asociados al dolor, a partir de la evaluación e indagación en las explicaciones de las enfermedades, alteraciones y trastornos que desequilibran la salud.

Inductores para determinar conocimientos esenciales y acciones preparatorias.

Los estudiantes deben precisar los aspectos psicológicos asociados al dolor y los síntomas y síndromes psíquicos

4) Tarjetas de estudio para el despliegue del proceso de intervención

Criterios: La prestación de los servicios de atención médica integral en forma oportuna y continua a la población, con énfasis en la prevención y modificación de los factores y conductas de riesgo; y la coordinación de acciones de educación para la salud, promoción de salud y profilaxis en el hombre sano, en interrelación con el medio.

Asignatura Psicología.

Objetivo: Identificar la sucesión finita de acciones que se requieren para hacer referencia a la existencia de recursos psicológicos interactivos que garanticen el afrontamiento de los problemas de salud comunitaria y la introducción de acciones psicológicas de promoción y prevención en salud, a partir de la coordinación y prestación de los servicios de atención médica integral.

Inductores para determinar conocimientos esenciales y acciones preparatorias.

Los estudiantes deben precisar respecto a la existencia de recursos psicológicos para el afrontamiento de los problemas de salud: ¿toma de conciencia?, ¿percepción de cambios físicos y hábitos cotidianos?, ¿estados psicológicos de mayor bienestar?, ¿población diana?, ¿técnicas de educación para la salud?

Asignatura Psicología Médica.

Objetivo: Identificar la sucesión finita de acciones que se requieren para hacer referencia a la inexistencia de recursos psicológicos interactivos que garanticen 
el afrontamiento de los problemas de salud comunitaria y la confección de historias psicosociales, a partir de la coordinación y prestación de los servicios de atención médica integral.

Inductores para determinar conocimientos esenciales y acciones preparatorias. Los estudiantes deben precisar respecto a la inexistencia de recursos psicológicos para el afrontamiento de los problemas de salud.

\section{Fase de ejecución}

Tareas docentes en respuesta a necesidades básicas de aprendizaje para la solución de problemas específicos de la profesión.

Las tareas docentes de la disciplina Psicología en respuesta a necesidades básicas de aprendizaje para la solución de problemas específicos de la profesión, derivan actividades que permiten la cumplimentación de acciones en los servicios de la asistencia sanitaria esencial ante el despliegue de los procesos de dispensarización, análisis de la situación de salud, diagnóstico e intervención. La tipología que se asume en las mismas responde a las carencias de fundamentación, interpretación y aplicación.

1) Tareas docentes de fundamentación con énfasis en la dispensarización y en el análisis de la situación de salud

Las tareas docentes de fundamentación con énfasis en la dispensarización y en el análisis de la situación de salud, tienen como objetivo que los estudiantes sean capaces de establecer bases teóricas y prácticas que proporcionen criterios sólidos para la argumentación y demostración de evidencias de aprendizaje afines a los referidos procesos - devenidos actividades - a concretar en los servicios de la asistencia sanitaria esencial.

Asignatura Psicología.

Trabajo independiente: Grupos de edades.

Objetivo: Fundamentar los aspectos básicos acerca del desarrollo humano en sus diferentes grupos de edades, propiciando la necesidad de afrontar el estudio de las personas humanas a través de su sistema de relaciones sociales. 
Precisiones metodológicas.

En actividades previas los estudiantes deben cumplimentar la elaboración independiente de las tarjetas de estudio referidas a los procesos de dispensarización y análisis de la situación de salud.

Se sugiere desarrollarla en la sexta semana lectiva del cuarto semestre académico, en el que se imparte la asignatura Psicología a los estudiantes de segundo año de la carrera de Medicina; momento en el que se profundizan los conceptos generales sobre el desarrollo humano, y sus etapas evolutivas.

En integración con la asignatura propia de la actividad del egresado Medicina Comunitaria, se orientará seleccionar una familia funcional para la ejecución de acciones referidas a:

- La confección de historias clínicas individuales con énfasis en el proceso de desarrollo psicológico del ser humano y en las características de las diferentes etapas del ciclo vital.

- La confección de la historia clínica familiar con énfasis en las demandas y exigencias del programa de atención a la salud familiar, y en las particularidades de la violencia intrafamiliar y su repercusión en la infancia.

- La confección de fichas epidemiológicas con énfasis en los niveles determinantes de la salud comunitaria y en el diagnóstico psicosocial del modo de vida familiar. Asignatura Psicología Médica.

Trabajo independiente: Relación médico-paciente-familia-comunidad en diversas circunstancias asistenciales.

Objetivo: Fundamentar los aspectos básicos de la relación médico-pacientefamilia-comunidad en diversas circunstancias asistenciales, favoreciendo al razonamiento de los principios generales de la ética médica.

Precisiones metodológicas.

En actividades previas los estudiantes deben cumplimentar la elaboración independiente de las tarjetas de estudio referidas a los procesos de dispensarización y análisis de la situación de salud.

Se sugiere desarrollarla en la sexta semana lectiva del quinto semestre académico, en el que se imparte la asignatura Psicología Médica a los estudiantes de tercer año 
de la carrera de Medicina; momento en el que se profundizan los conceptos generales sobre el estrés psicológico y su repercusión en el proceso salud-enfermedad.

En correspondencia con la asignatura propia de la actividad del egresado Propedéutica Clínica y Semiología Médica, se orientará seleccionar un paciente sujeto a prescripciones diagnósticas y terapéuticas para la ejecución de acciones referidas a:

- La confección de la historia clínica individual con énfasis en los factores psicosociales que favorecen y/o entorpecen el proceso de comunicación en salud. - La confección de la historia clínica familiar con énfasis en los factores que influyen en la afectación de la salud familiar y en los efectos del estrés ante la experiencia de tratamiento con ingreso hospitalario de un integrante de la familia.

- La confección de fichas epidemiológicas con énfasis en las alteraciones psíquicas que integran el cuadro interno de la enfermedad en el paciente seleccionado y en las afectaciones a la salud de cada uno de los integrantes de su familia.

2) Tareas docentes de interpretación con énfasis en el diagnóstico

Las tareas docentes de interpretación con énfasis en el diagnóstico, tienen como objetivo que los estudiantes sean capaces de explicar elementos, relaciones y razonamientos, que faciliten la comprensión y expresión de evidencias de aprendizaje afines al referido proceso -devenido actividad- a concretar en los servicios de la asistencia sanitaria esencial.

Asignatura Psicología.

Trabajo independiente: La personalidad, formación y desarrollo, componentes estructurales e influencia en el proceso salud-enfermedad.

Objetivo: Interpretar los aspectos básicos del desarrollo de la personalidad, los componentes que la estructuran y su influencia en el proceso salud-enfermedad, propiciando la necesidad de afrontar el estudio de las personas humanas a través de su sistema de relaciones sociales.

Precisiones metodológicas.

En actividades previas los estudiantes deben cumplimentar la elaboración independiente de la tarjeta de estudio referida al proceso de diagnóstico.

Se sugiere desarrollarla en la novena semana lectiva del cuarto semestre académico, en el que se imparte la asignatura Psicología a los estudiantes de 
segundo año de la carrera de Medicina; momento en que se profundizan conceptos relacionados con la personalidad, su formación y desarrollo, los componentes estructurales y su influencia en el proceso salud-enfermedad.

En correspondencia con la asignatura propia de la actividad del egresado Medicina Comunitaria, se orientará seleccionar una familia funcional para la ejecución de acciones referidas a:

- La evaluación del funcionamiento de los procesos psicológicos en cada uno de los integrantes de la familia seleccionada.

- La indagación sobre las características de los componentes estructurales de la personalidad en cada uno de los integrantes de la familia seleccionada.

Asignatura Psicología Médica.

Trabajo independiente: Los aspectos psicológicos del estrés asociados al dolor y las alteraciones psíquicas que desequilibran la salud en su expresión individual y familiar.

Objetivo: Interpretar los aspectos psicológicos del estrés asociados al dolor y las alteraciones psíquicas que desequilibran la salud, propiciando la necesidad de afrontar el estudio de las personas humanas a través de su sistema de relaciones sociales.

Precisiones metodológicas.

En actividades previas los estudiantes deben cumplimentar la elaboración independiente de la tarjeta de estudio referida al proceso de diagnóstico.

Se sugiere desarrollarla en la novena semana lectiva del quinto semestre académico, en el que se imparte la asignatura Psicología Médica a los estudiantes de tercer año de la carrera de Medicina; momento en el que se profundizan los aspectos psicológicos del estrés asociados al dolor, así como las alteraciones psíquicas que desequilibran la salud en su expresión individual y familiar.

En correspondencia con la asignatura propia de la actividad del egresado Propedéutica Clínica y Semiología Médica, se orientará seleccionar un paciente sujeto a prescripciones diagnósticas y terapéuticas para la ejecución de acciones referidas a:

- La evaluación de los aspectos psicológicos asociados al dolor que caracterizan el cuadro actual de salud del paciente seleccionado. 
- La indagación sobre las alteraciones psíquicas vinculadas al estrés que se evidencian tanto en el paciente seleccionado como en los integrantes de su familia.

3) Tareas docentes de aplicación con énfasis en la intervención

Las tareas docentes de aplicación con énfasis en la intervención, tienen como objetivo que los estudiantes sean capaces de llevar a la práctica determinados procedimientos teóricos y metodológicos que propicien mediaciones efectivas, afines al referido proceso - devenido actividad- a concretar en los servicios de la asistencia sanitaria esencial.

Asignatura Psicología.

Trabajo independiente: Conceptos básicos, sistema de relaciones categoriales y principales medios de interacción social del médico general en la comunidad.

Objetivo: Aplicar los conocimientos sobre el sistema de relaciones categoriales y los principales medios de interacción social del médico general en la comunidad, favoreciendo al razonamiento de los principios generales de la ética médica.

Precisiones metodológicas.

En actividades previas los estudiantes deben cumplimentar la elaboración independiente de la tarjeta de estudio referida al proceso de intervención..

Se sugiere desarrollarla en la oncena semana lectiva del cuarto semestre académico, en el que se imparte la asignatura Psicología a los estudiantes de segundo año de la carrera de Medicina; momento en el que se profundizan los conceptos generales sobre el sistema de relaciones categoriales y los principales medios de interacción social del médico general en la comunidad.

En correspondencia con la asignatura propia de la actividad del egresado Medicina Comunitaria, se orientará seleccionar una familia funcional para la ejecución de acciones referidas a:

- La determinación de la existencia de recursos psicológicos interactivos, que garanticen el conocimiento y afrontamiento de los problemas de salud en la familia seleccionada.

- La introducción de acciones psicológicas de prevención y promoción en salud, que incluyan a cada uno de los integrantes de la familia seleccionada.

Asignatura Psicología Médica. 
Trabajo independiente: Principales elementos de psicodiagnóstico clínico y psicoterapia útiles para el alivio, la curación y la rehabilitación en la práctica médica. Objetivo: Aplicar los conocimientos sobre los principales elementos de psicodiagnóstico clínico y psicoterapia, útiles para el alivio, la curación y la rehabilitación en la práctica médica, favoreciendo al razonamiento de los principios generales de la ética médica.

Precisiones metodológicas.

En actividades previas los estudiantes deben cumplimentar la elaboración independiente de la tarjeta de estudio referida al proceso de intervención.

Se sugiere desarrollarla en la oncena semana lectiva del quinto semestre académico, en el que se imparte la asignatura Psicología Médica a los estudiantes de tercer año de la carrera de Medicina; momento en el que se profundizan los principales elementos de psicodiagnóstico clínico y psicoterapia útiles para el alivio, la curación y la rehabilitación en la práctica médica.

En correspondencia con la asignatura propia de la actividad del egresado Propedéutica Clínica y Semiología Médica, se orientará seleccionar un paciente sujeto a prescripciones diagnósticas y terapéuticas para la ejecución de acciones referidas a:

- La determinación de la inexistencia de recursos psicológicos interactivos, que garanticen el conocimiento y afrontamiento de los problemas de salud en el paciente seleccionado.

- La confección de la historia psicosocial del paciente seleccionado.

Tránsito por una secuencia de etapas en las que se produce la apropiación del contenido del proceso de formación

1) Etapa de familiarización

Esta etapa corresponde a los estudiantes de segundo año de Medicina. Su principal objetivo es propiciar un primer acercamiento a la actividad de atención psicológica integral que se desarrolla en las diferentes instituciones de la asistencia sanitaria esencial y la consolidación de las potencialidades educativas adquiridas a través del componente académico en las asignaturas propias de la actividad del egresado. 
Se hace énfasis en la observación y el análisis de estrategias desarrolladas por los profesionales de la salud donde se realice la educación en el trabajo. También se incluye el estudio de las condiciones socioeconómicas que pueden favorecer o entorpecer el proceso salud-enfermedad. Además se planificarán actividades colectivas de prevención y promoción en salud dirigidas a individuos potencialmente sanos, como niños y adolescentes, con la intención de reforzar factores de protección.

2) Etapa de iniciación.

Esta etapa corresponde a los estudiantes de segundo año de Medicina. Durante la misma se consolidan los conocimientos y habilidades desarrollados desde el primer año de la carrera que aseguran la efectividad del proceso de intervención psicosocial. Se desarrollarán actividades de prevención y promoción en salud de acuerdo a los factores de riesgo existentes.

El énfasis de las actividades estará en lograr que los estudiantes establezcan una adecuada relación entre estilo de vida, condiciones de vida, calidad de vida, modo de vida e indicadores de predisposición a la enfermedad. Se incluyen en esta etapa las actividades de caracterización comunitaria con énfasis en factores de riesgo y necesidades educativas, planificación y ejecución de acciones de prevención y promoción en salud, así como la evaluación del impacto inmediato de las acciones desarrolladas. Se llevarán a cabo en la clínica o en instituciones de la comunidad bajo la dirección de un profesional de la psicología.

3) Etapa de consolidación y generalización.

Esta etapa corresponde a los estudiantes de tercer año de Medicina. La misma debe posibilitar la consolidación y generalización de los conocimientos y habilidades adquiridos durante las etapas anteriores, con los conocimientos y habilidades de la asignatura Psicología Médica. El objetivo de esta etapa es potenciar en los estudiantes habilidades para la realización del diagnóstico clínico y la intervención psicoterapéutica en personas sanas o en riesgo de enfermar.

El sistema de actividades incluye el diagnóstico de salud, las actividades educativas individuales y familiares a partir del diagnóstico clínico realizado, las actividades colectivas de prevención y promoción en salud, así como actividades de evaluación inmediatas y a mediano plazo. 
4) Etapa de sistematización e integración.

Esta etapa corresponde a los estudiantes de cuarto, quinto y sexto años de Medicina. Les corresponde la ejecución de tareas de prevención y promoción en salud, caracterización de la salud individual y colectiva de la población, detección de factores de riesgos ambientales, trabajo en equipo con los especialistas y atención médica de urgencia.

Durante el desarrollo de su práctica preprofesional y a partir del diagnóstico de salud individual y colectiva de su área asistencial, los estudiantes de estos últimos años de la carrera de Medicina deben desarrollar una intervención educativa dirigida a atender uno o más problemas de salud comunitaria; dicho plan de acción implica la participación de instituciones comunitarias y abarca la evaluación del impacto de las acciones desarrolladas a mediano y largo plazo.

\section{Fase de control}

1) Evaluación sistemática

La evaluación sistemática del proceso de asimilación de las bases orientadoras de las acciones considerará que los estudiantes:

- Entren en contacto con la explicación de los aspectos esenciales que integran los esquemas de bases orientadoras para la realización de las acciones.

- Contribuyan y/o participen en la elaboración de las tarjetas de estudio.

- Asimilen el contenido operacional de las acciones mediante su empleo en la solución de tareas docentes que respondan a problemas de la profesión.

- Intercambien roles en la explicación en voz alta de soluciones a las tareas docentes y el control de lo expresado, registrándose los errores.

2) Interioricen de forma abreviada y a través del lenguaje interno las acciones. Evaluación de los resultados en etapas intermedias y finales

Se trata de evaluar los resultados obtenidos en las etapas intermedias y finales del proceso de formación, así como su contrastación con los objetivos planteados y con el diagnóstico realizado. 
Durante su implementación, el colectivo pedagógico de la disciplina Psicología debe considerar los impactos educativos de la estrategia pedagógica en la formación del modo de actuación atención médica integral.

\section{Conclusiones}

1. La formación del modo de actuación atención médica integral desde la disciplina Psicología precisa una lógica al accionar en la actividad pedagógica donde las funciones afines al perfil del profesional se estructuran en invariantes de habilidades y conocimientos, para la realización de acciones específicas que respondan a las acciones generalizadas de dispensarización, análisis de la situación de salud, diagnóstico e intervención;

2. La estrategia pedagógica integra fases, direcciones e indicaciones que implican la determinación de los esquemas de bases orientadoras para la creación de las condiciones que intervienen en el proceso de formación de las acciones a concretar a través del componente laboral y en interacción con los componentes académico e investigativo, a partir de la cumplimentación con apoyo en las tarjetas de estudio de un sistema de tareas docentes dirigido a solucionar los principales problemas de la profesión.

\section{Referencias}

GALPERIN, P. Ya. Stage-by-Stage formation as a Method of Psychological Investigation. Journal of Russian and East European Psychology, Philadelphia, v. 30, n. 4, p. 60-80, 1992a.

GALPERIN, P. Ya. The Problem of Activity in Soviet Psychology. Journal of Russian and East European Psychology, Philadelphia, v. 30, n. 4, p. 37-59, 1992b.

HORRUITINER SILVA, P. La universidad cubana: el modelo de formación. La Habana: Félix Varela, 2006.

MINSAP. Plan de Estudio de la carrera de Medicina [CD-ROM]. La Habana: Universidad de Ciencias Médicas, 2014. 
TALÍZINA, N. Conferencias sobre "Los Fundamentos de la Enseñanza en la Educación Superior". Universidad de La Habana: Departamento de Estudios para el Perfeccionamiento de la Educación Superior, 1985. https://doi.org/10.22201/iisue.20072872e.2012.8.71

TALÍZINA, N. Psicología de la enseñanza. Moscú: Progreso, 1988.

TOLEDO MÉNDEZ, M. A.; CABRERA RUIZ, I. I Práctica sistemática de la formación laboral en atención médica integral desde la asignatura Psicología I. Revista de Educación Médica Superior, La Habana, v. 30, n. 4, 2016c. Disponible en: http://www.ems.sld.cu/index.php/ems/article/view/818.

Recebido em abril de 2020. Aprovado em julho de 2020. 